\title{
LA PROBLÉMATIQUe LinguistiQue de L'EXPRESSION À GENÈVE ENTRE 1900 ET 1940 : \\ Charles Bally, Albert Sechehaye et Henri Frei
}

\author{
Anamaria Curea \\ Université Babeş-Bolyai de Cluj-Napoca
}

\begin{abstract}
Résumé
À partir d'une analyse de la composante théorique des productions scientifiques de Charles Bally, Albert Sechehaye et Henri Frei entre 1900 et 1940, nous nous proposons de relever et d'analyser l'effet d'homogénéité, possible trait identitaire de l'école, qui se dégage autour de la catégorie de l'expression (comportant deux volets, expressivité et exprimabilité). Cette problématique développée par les trois linguistes genevois permet d'identifier quelques points de convergence significatifs, liés principalement à trois domaines de représentation communs : le statut de la psychologie, l'approche de l'affectivité et les modes de représentation du sujet parlant. Dans la seconde partie de notre article, en évoquant les notions de conformité et de variation (Schlanger 2008), nous examinons les modes d'articulation entre ces points de convergence et un autre trait identitaire commun, qualifié de « saussurisme minimal » (Godel 1961 et Amacker 1975).
\end{abstract}

\section{Mots-clés}

Bally, conformité, École linguistique de Genève, expression, Frei, identité, Saussure, Sechehaye, unité, variation

\section{Abstract}

This article takes as its starting point an analysis of the theoretical component of the scientific programs conceived by Charles Bally, Albert Sechehaye and Henri Frei between 1900 and 1940. Our purpose is to point out and examine the homogeneity effect, possibly an identifying trait of the school, which comes to light when dealing with the expression category. This linguistic issue, which is treated by the three linguists from Geneva, brings to the fore some significant points of convergence, linked primarily to three common domains of representation: the status of psychology, the approach to affectivity and the ways in which the speaking subject may be envisaged. In the second part of the article, by bringing forward the notions of conformity and variation (Schlanger 2008), we discuss the links between these points of convergence and another common identifying trait, described as "minimal Saussureanism" (Godel 1961 et Amacker 1975).

\section{Keywords}

Bally, conformity, expression, Frei, Geneva School of Linguistics, identity, Saussure, Sechehaye, unity, variation 
Cette réflexion s'appuie sur quelques éléments que l'analyse du composant théorique (Auroux 1998, p. 8) des travaux de Charles Bally, Albert Sechehaye et Henri Frei, publiés entre 1900 et 1940, apporte à la discussion sur l'identité de l'école de Genève. Quels traits identitaires caractérisent l'école avant 1940 ? Estce que l'originalité manifeste de chacun des trois membres empêche l'émergence d'un ensemble idéologique cohérent ? L'ensemble des " positions saussuriennes » (Godel 1984 [1961], p. 78) qu'ils adoptent suffit-il à assurer une homogénéité audelà de la diversité de leurs entreprises ? Comment caractériser leur saussurisme ? Et enfin, quelles notions mobiliser pour une possible définition du concept d'école linguistique?

\section{Pour Une PROBlÉMATIQUe LINGUISTIQUe DE L'EXPRESSION}

Nous cherchons à déterminer l'existence d'éléments théoriques ou de nœuds conceptuels autour desquels pourrait se coaguler une dynamique des idées, apte à instituer un objet partagé, qui, pour être convenu et discuté, n'en est pas moins disputé. La problématique linguistique de l'expression, différemment envisagée par chacun des trois linguistes, pourrait révéler quelques points de convergence significatifs, qui produisent un effet d'homogénéité dans la définition de ce domaine notionnel. Trois axes communs se dessinent autour du statut de la psychologie, du rôle de l'affectivité et des modes de conceptualisation du sujet parlant.

Si ces trois axes peuvent être envisagés comme les coordonnées de trois domaines de représentation communs, jusqu'à quel point leurs représentations proprement dites se rapprochent-elles, quelle étendue ces domaines occupent-ils dans l'ensemble de leurs travaux entre 1900 et 1940, comment s'institue cet objet de partage au sein de l'école ? Et quel genre d'objet de partage est la problématique de l'expression?

\subsection{Expression, expressivité, exprimabilité. Comment se décline la problématique linguistique de l'expression à Genève entre 1900 et 1940 ?}

Chez Bally, la problématique de l'expression fait l'objet d'une approche évolutive, marquée par trois moments de sa production scientifique : ses travaux de stylistique, son article de 1926 intitulé « Le mécanisme de l'expressivité linguistique », publié dans Le langage et la vie (ci-après $L V$ ), et son ouvrage de 1932, Linguistique générale et linguistique française (ci-après $L G L F$ ). Ce parcours est jalonné par quelques aménagements terminologiques et conceptuels.

Dans la stylistique, la notion d'expression sert à définir un nouveau champ disciplinaire. Située au cœur du système explicatif, elle est peu stable et subit un glissement, du fait de se voir attribuer une extension variable. Le terme expressif 
oscille entre deux acceptions. D'une part, il est associé au domaine de l'expression, envisagée comme champ de manifestation des rapports entre la pensée et la langue (« système expressif », « moyens d'expression », « faits d'expression ») son extension maximale -, d'autre part, son domaine est progressivement restreint à l'ensemble des contenus affectifs des faits d'expression, qui ne peuvent être relevés que par contraste avec le domaine opposé, celui des facteurs intellectuels. Cette seconde acception fait coïncider l'expressif et l'affectif, et détermine la conception qu'a Bally de la langue parlée, essentiellement affective. Dès lors, l'extension variable du domaine de la notion d'expression s'associe à un dédoublement de l'enjeu de la stylistique.

La stylistique se serait ainsi en quelque sorte détournée de son enjeu initial, celui d'une science générale de l'expression, pour se concentrer sur l'étude des valeurs affectives du « langage de la vie et de l'action ». Significativement, ce glissement est opéré au moment où Bally entreprend l'analyse proprement dite des moyens d'expression par la méthode stylistique. Dans ses analyses des moyens d'expression lexicaux, prosodiques ou syntaxiques, du fait de se rapporter constamment aux éléments affectifs, la portée ou l'incidence de la notion d'expression se restreint jusqu'à devenir synonyme d'affectivité et de subjectivité.

Une nouvelle étape dans la conceptualisation de 1'expressif par Bally est marquée par son article de 1926, « Mécanisme de l'expressivité linguistique » et dans $L G L F$ de 1932. Dans la stylistique, la problématique de l'expressif est associée, d'un côté, au projet d'une théorie générale de l'expression et, d'un autre côté, à la nécessité de fixer un critère de classement des données empiriques. Dans $L V$ et $L G L F$, cette problématique est en quelque sorte sauvée par le traitement plus abstrait de l'expressivité comme "mécanisme », ainsi que par le rôle qui lui est attribué dans l'évolution même d'une langue (le « changement linguistique », la « vie du langage »). En envisageant un mécanisme de l'expressivité, Bally semble dépasser la principale critique apportée par Saussure', à savoir l'impossibilité de concevoir une étendue dans le langage pour ce qui est affectif. Son objectif n'est plus de fixer une étendue à l'affectif, mais d'envisager un mécanisme apte à rendre compte de la complexité d'un état de langue et du changement linguistique.

Si pour Bally, la problématique de l'expression est la clé de la construction d'un projet disciplinaire, pour Sechehaye, elle est la clé d'une explication raisonnée de la complexité de l'objet linguistique, et de la réforme de la grammaire. C'est une science de l'expression grammaticale qu'il envisage de fonder (Sechehaye 1908a et 1908 b), avec beaucoup d'égards pour la propriété des notions utilisées ainsi que pour leur adaptation au type d'explication apportée. À son sens, le problème de

1 Cette critique est formulée dans une lettre du 3 juillet 1904 adressée par Saussure à Bally, reproduite dans CFS 48, 1994/1995, p. 96-97. 
l'expression est un problème d'exprimabilité plutôt qu'un problème d'expressivité (au sens de Bally). À l'intérieur de la problématique de l'expression, nous associons l'exprimabilité au traitement du problème du rapport entre la pensée et la langue, et l'expressivité à l'approche des manifestations linguistiques de l'affectivité. Le problème grammatical doit être posé en accord avec la loi de solidarité entre le moyen d'expression (la langue) et la chose exprimée (la pensée). Pour Sechehaye, le système de signes est organisé du point de vue matériel de façon à correspondre au « système abstrait de signes et de relations » (Ibid., p. 34). Il s'agit donc, comme pour Bally, d'une forme de mentalisme, mais c'est un mentalisme inséparable du formalisme foncier de la langue. Aucun des deux systèmes ne préexiste à l'autre, ils sont solidaires et ils fonctionnent conjointement, en parfaite interdépendance et simultanéité 2 .

La langue expressive, objet de la stylistique, conçue par Bally comme une zone intermédiaire entre le langage organisé et le langage de la vie, est envisagée par Sechehaye comme l'ensemble des actes de parole, où l'on peut identifier les éléments relevant de la grammaire ou de la convention, et les éléments expressifs naturels, qui ont des causes psychiques et s'adressent à l'intuition. Il établit une distinction entre l'expression grammaticale de la pensée et les éléments expressifs naturels relevant de la libre spontanéité du sujet. À son sens, l'intervention de l'intuition dans l'acte de parole caractérise la vie psychologique générale de l'individu, et l'intelligence intervient notamment dans le fonctionnement de l'organisme grammatical, dont elle assure l'existence. L'intuition est donc associée aux éléments extragrammaticaux, alors que l'intelligence institue l'élément grammatical (Sechehaye 1908, p. 70 et suiv.).

Chez Frei, dans La grammaire des fautes (1929) (ci-après GF), la problématique de l'expression se situe au croisement d'une idée de Bally, son maître, sur l'antinomie entre la communication et l'expression, et d'une autre, originale, qu'il avait maintenue malgré les réserves de son directeur (qui lui préférait la notion de «tendances »), sur l'existence de « besoins » en fonction desquels les procédés de la langue peuvent être classés et expliqués. Dans ce contexte, les idées de Frei présentent des similarités et des différences par rapport à la conception de Bally. Les similarités les plus immédiates concernent le recours à l'antinomie de la communication et de l'expression, la distinction entre processus et procédé, pour

2 Les deux approches théoriques associent l'expression et l'expérience perceptive. Pourtant, Bally tend à attribuer une existence formelle aux éléments affectifs, alors que Sechehaye insiste sur la solidarité totale entre les deux plans de l'expression, l'exprimant et l'exprimé. Dans leur ample article de 2008 sur les modèles et pensées de l'expression, dans une perspective microgénétique, Rosenthal et Visetti (2001, p. 188) estiment qu' « il est inscrit dans la structure même de l'expression (au sens fort), que l'on tende à fétichiser la manifestation $\mathrm{Y}$, en la détachant du moment expressif, et en la rabattant sur quelque versant « extérieur» (par exemple en la retraitant comme une morphologie indépendante) ». 
distinguer affectivité fortuite et expressivité, le traitement de la figuration, l'opposition entre l'expressivité et l'arbitraire. D'autre part, chez Frei se manifeste un intérêt plus marqué pour une systématisation de cette problématique (sous-types, classement des procédés) et pour l'explicitation du rapport entre l'expressivité et le degré de conscience du sujet parlant dans l'usage de la langue.

Les conceptualités constitutives de la problématique de l'expression, surtout chez Bally et Sechehaye, et dans une moindre mesure, chez Frei, présentent quelques points de convergence et un degré d'affinité qui permettent de repérer trois axes ou domaines de représentation, envisagés différemment par les trois linguistes : la place de la psychologie, le rôle de l'affectivité, et le statut du sujet parlant. Les deux premiers axes ont un rapport étroit au troisième, avec lequel ils forment un ensemble autour de l'idée que la langue et le sujet sont inséparables.

\subsection{Domaines et modes de représentation}

\subsubsection{La psychologie dans la linguistique}

Dans les travaux de stylistique de Bally ${ }^{3}$, il est possible d'identifier deux catégories de références à la psychologie : la psychologie comme domaine scientifique et la psychologie comme méthodologie générale.

La première classe de références à la psychologie est relativement restreinte. Quelques noms de psychologues allemands sont brièvement évoqués dans le Précis de 1905 (note 1, p. 127) : Berthold Delbrück (Grundfragen der Sprachforschung 1901), Wilhelm Wundt (Völkerpsychologie 1900) et Hermann Paul (Prinzipien der Sprachgeschichte 1898). Dans ses notes plutôt lapidaires, Bally reconnaît l'influence de ces psychologues qui ont examiné les bases psychologiques de l'étude du langage.

La seconde catégorie de références à la psychologie est particulièrement riche : psychologie expérimentale, psychologie pratique, psychologie sociale, psychologie de la valeur. Quel sens attribuer à cette profusion de qualifications ? Loin de les considérer comme des branches de la psychologie, Bally semble les envisager comme coextensives de la linguistique. La psychologie semble désigner ici une méthodologie générale dans l'étude linguistique, étant donné que pour Bally, le langage est un « phénomène naturel », régi par des lois « sociales » et « psychologiques ». L'objet lui-même étant psychologique, la méthode la plus appropriée à son étude présente, par voie de conséquence, une dimension générale psychologique ${ }^{4}$.

3 Dans son Rapport sur la création d'une chaire de stylistique, Saussure associait la stylistique à « l'explication des formules de la langue en tant que motivées par tel ou tel état psychologique » (Saussure 2002, p. 272-273).

4 Voir B. Colombat, J.-M. Fournier et C. Puech (2010, p. 212) sur les sens de la généralité dans la linguistique, dont le troisième désigne « une sorte de métissage des points de vue, 
Cette conception de la psychologie comme méthodologie générale dans l'étude des données linguistiques explique l'instabilité qui caractérise l'usage de ce terme. Parfois, tout le domaine de la linguistique ou de la stylistique est assimilé à la psychologie $\mathrm{e}^{5}$. Ailleurs, des distinctions sont proposées entre ces disciplines, à partir d'un rapprochement variable des faits étudiés par rapport aux formes de la pensée ou aux formes de la langue ${ }^{6}$.

Sechehaye envisage avant tout la linguistique comme une science de l'Homme, intégrée au programme d'une anthropologie générale (Sechehaye 1908b, p. 17). La recherche d'une méthode rationnelle pour la science du langage l'amène à une restructuration des champs disciplinaires à partir d'un principe unique. Il définit le langage comme un phénomène total et comme une activité psychique qui se réalise par l'intermédiaire de l'organisme. En tant que telle, cette activité demande à être étudiée de plusieurs points de vue, qui se réclament de la physiologie, de la logique et de la psychologie.

La nature psychologique du langage est expliquée par deux particularités du sujet parlant, l'automatisme grammatical et la libre spontanéité. À son sens, l'organisme grammatical ne peut à lui seul former un langage et c'est là qu'interviennent les facteurs de nature psychologique. La réalisation de la parole par un acte de volonté et d'intelligence réclame également des éléments relevant de la libre spontanéité du sujet parlant. Certains accents, les nuances de l'intonation, l'ordre des mots sont régis par une nécessité psychologique. Extérieurs à la convention, qui n'exerce aucune influence dominante sur eux, ces éléments déterminent la compréhension du discours du fait que leur interprétation repose sur l'intuition de leurs causes psychiques (Sechehaye 1908, p. 52).

La nature de l'objet amène Sechehaye à isoler un élément relevant de la convention, appelé élément grammatical, et un autre type d'éléments, dont la présence est motivée par la nécessité psychologique, les éléments extragrammaticaux. Pour prolonger ce mode de représentation, le linguiste affirme la nécessité de distinguer entre deux types de «psychologie » » : une psychologie collective, dont relève l'élément conventionnel ou grammatical, et une psychologie individuelle ou simple, dont se réclament les éléments extragrammaticaux (Sechehaye 1983 [1908a], p. 184 et suiv. et 1908b, p. 53).

sociologique et psychologique, tout particulièrement, sur le langage ».

5 La théorie de l'expression est assimilée à la « face linguistique de la psychologie de la valeur » (Bally 1911, p. 116).

6 Bally (1914, p. 265-268) propose une approche originale, fondée sur le passage progressif des « faits de pensée » aux « faits d'expression », à travers quatre étapes, chacune faisant l'objet d'une discipline différente : la psychologie, la psychologie du langage, la stylistique, la grammaire. 
Là où Bally opposait l'intelligence à l'affectivité, Sechehaye distingue intelligence et intuition, convention et nature. L'emboîtement du langage grammatical dans le langage prégrammatical entraîne l'emboîtement de la psychologie collective dans la psychologie individuelle ou physiologique. La psychologie collective est une science de l'Homme et de son activité psychologique dans la mesure où elle est régie par les nécessités de la vie commune. Sa méthode est déductive et envisage l'effort intellectuel qui aboutit à la création de la grammaire à travers la nécessité de comprendre et d'être compris, à travers la socialité et l'intersubjectivité (Sechehaye 1908b, p. 53).

Chez Frei, on constate plutôt une tendance à éviter la problématique des rapports entre la linguistique et la psychologie. Sa linguistique fonctionnelle n'est pas, il l'affirme explicitement, un retour à l' « ancienne psychologie du langage » (Frei 2007 [1929], p. 44). L'étude des fautes, type de comportement langagier qui offre les indices de certains besoins régissant tout le fonctionnement du langage, généralement valables dans toutes les langues, se refuse aux explications en termes de causes psychiques ou psychologiques. Dans la perspective finaliste qui caractérise la $G F$, les besoins sont déclenchés par des déficits, et engendrent des procédés, mais il n'est pas question de nécessité psychologique. Frei s'intéresse moins aux causes psychiques du fonctionnement de la langue qu'à la fonction de certains phénomènes qui transgressent la norme de la langue, aux besoins dont ils sont les indices, au classement des faits linguistiques en fonction de ces besoins. La psychologie, qu'elle soit conçue comme une science ou comme une méthode, ne s'inscrit pas dans ce cadre-là.

\subsubsection{Le statut de l'affectivité}

Pour Bally, la problématique de l'affectivité est d'une importance capitale. Déjà dans le Précis, plusieurs tentatives convergent vers la représentation du langage comme champ où se combinent deux types d'éléments, un type « intellectuel » et un type « affectif », dont l'étude réclame une méthode descriptive et expérimentale permettant d'étudier les effets des faits de langue sur la sensibilité du sujet parlant ${ }^{7}$.

7 Dans une lettre adressée à Bally en 1904 à propos du Précis, Saussure affirmait que l'importance comparative attribuée à la distinction intellectuel/affectif n'était pas suffisamment justifiée, et qu'elle ne parvenait pas à fixer pour l'affectif « telle ou telle étendue dans l'ensemble des phénomènes ». À son tour, dans son article de 1908a, Sechehaye apporte une critique nuancée de cette distinction, et propose une approche différente, plus pertinente, à son sens. Au lieu d'opposer l'intellectuel à l'affectif, il envisage plutôt l'existence d'un élément conventionnel et d'un élément naturel ou spontané. Plus tard, en 1939, il estime que la division entre l'intellectuel et l'affectif est justifiée, à condition de servir à la division de la grammaire en deux chapitres différents par leur objet et par leur méthode : le structuralisme et la grammaire affective. 
La définition de la stylistique de 1909 évoque d'abord la sensibilité et le contenu affectif. Cette nouvelle science étudie « les faits d'expression du langage organisé du point de vue de leur contenu affectif », parcours qui va dans les deux sens : l'action des faits de langage sur la sensibilité et l'expression de la sensibilité par les faits de langage (Bally 1951 [1909], p. 16). En 1910, Bally redéfinit

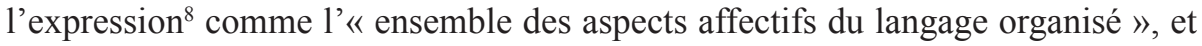
la stylistique comme l'étude de ces aspects affectifs et des procédés linguistiques qui servent à les produire (Bally 2007 [1910], p. 100). Sous l'influence de sa vision vitaliste, ses idées sur l'affectivité et la subjectivité dans le langage changent dans $L V$ de 1926 et dans $L G L F$ de 1932. L'affectivité est associée, comme dans la stylistique, au langage de la vie et de l'action, ainsi qu'à l'expression du sentiment et de l'émotion du sujet parlant, mais elle n'est plus considérée comme un critère de classement des données, ni comme un attribut du contenu d'un fait de langue (le « contenu affectif»). Elle est en revanche définie comme manifestation naturelle et spontanée des formes subjectives de la pensée, elle est envisagée dans ses rapports avec les désirs, les sensations, les volontés et les jugements de valeur (Bally 1977 [1926], p. 75).

Chez Sechehaye, l'intérêt pour le langage affectif est, comme chez Bally, un des axes de la problématique de l'expression, qui prend pour lui la forme d'une science de l'expression grammaticale. La différence la plus notable entre son approche et celle de son collègue réside dans sa décision de reléguer l'affectif hors de la grammaire, et de lui attribuer une fonction psychologique plutôt que linguistique.

Dans son Programme (1908), Sechehaye introduit le principe d'emboîtement pour opérer une division de l'objet de la linguistique ainsi que de la linguistique elle-même. Il identifie ainsi deux formes de langage : le langage affectif et le langage grammatical ou organisé, le second étant emboîté dans le premier. Présente dans l'expression instinctive des émotions, régie par des lois psychologiques, cette forme de langage défie toute tentative d'organisation. Il faudrait l'envisager, selon Sechehaye, comme une «modalité du sujet» (Ibid., p. 81), animé par une nécessité psychologique, visée qui associe le caractère expressif des signes à l'interprétation intuitive de l'impression qu'ils produisent sur la sensibilité ou sur l'imagination d'autrui. Ces signes n'expriment clairement aucune idée, mais ils sont provoqués par les idées, qu'ils accompagnent. Le langage affectif est à la fois plus général et plus individuel que le langage organisé, car il s'explique par la même constitution psycho-physique des individus, qui sont pourtant capables d'y imprimer

8 En éditant une lettre de Bally à Sechehaye de 1908 où il est question du lien entre la stylistique et l'expression, A.-M. Fryba-Reber (2001, p. 432-433) signale que le mot expression est d'abord biffé, puis souligné en pointillé. 
une marque originale. L'affectif se situe donc, à son sens, à l'extérieur de la grammaire, il ne peut donc pas être une propriété de la langue.

La grammaire possède, selon Sechehaye, la propriété fondamentale de ne pas supprimer les mouvements de la vie affective, mais de les réclamer. Inversement, les impulsions psychologiques n'empiètent pas sur le fonctionnement grammatical, grâce à l'activité intellectuelle. Comme chez Bally, mais selon un principe différent, les deux formes de langage se combinent dans des proportions variables, la parole relevant soit d'un facteur affectif dominant, soit d'un facteur intellectuel dominant. Le langage affectif a la propriété de ne pas exister sous forme pure, ce que l'on étudie sous cette dénomination ce sont les faits de langage qui tendent vers l'expression affective. Aucune des deux formes de langage n'existe à l'état pur. Le langage affectif a l'unique propriété de pouvoir évoluer vers l'organisation grammaticale, sous l'action de l'intelligence, ou de rétrograder vers les formes inférieures de la vie psychique, sous le coup d'une forte émotion. En tant que telle, cette forme de langage est envisagée par Sechehaye comme une forme « insaisissable », « transitoire » et « instable » de l'activité psychique (Sechehaye 1908b, p. 91).

Le rôle de l'affectivité dans la linguistique fonctionnelle de Frei est relativement restreint. Si l'antinomie de l'expression et de la communication est reprise à son directeur de thèse et approfondie de manière originale, l'affectif est loin d'occuper la même place.

À propos de l'affectivité, Frei introduit une distinction nécessaire à une sélection pertinente des faits linguistiques classés comme expressifs. Ce critère est l'intention du sujet parlant. Il définit d'abord l'affectivité fortuite, qui est dégagée à l'insu du locuteur ou malgré lui, par la situation, puis un autre type d'affectivité, que le locuteur cherche à transmettre à son interlocuteur. Il réserve le nom d'expressivité au second type d'affectivité (Frei 2007 [1929], p. 302-303). La différence entre les deux types réside dans le degré d'implication de la conscience du sujet parlant. Seule l'affectivité résultant de l'exercice conscient de la volonté de transmettre un contenu émotif peut être appelée expressivité. L'affectivité fortuite est du ressort de la causalité, alors que l'expressivité est définie par la finalité. Frei adopte un point de vue qui s'appuie sur deux particularités du sujet parlant, son intention de communication et sa conscience, pour définir l'expressivité. La finalité dans la langue est ainsi une fonction du sujet parlant.

\subsubsection{Figure(s) du sujet parlant dans la problématique de l'expression}

Les deux axes que nous avons identifiés contribuent à la mise en place d'une perspective qui privilégie la relation entre le sujet et la langue. Cette caractéristique s'avère l'une des plus significatives pour la spécificité de l'école de Genève entre 1900 et 1940. Par leur intérêt pour la langue parlée, pour la variation dans la langue, 
pour les rapports entre la pensée et la langue, ces linguistes cherchent à instituer le sujet dans la langue à travers la problématique de l'expression.

La stylistique de Bally se caractérise par une hiérarchisation des facultés psychiques en fonction de leur degré d'activation dans la langue parlée. Le « moi » est ainsi associé à la part que « nous » prenons aux choses, à l'élément subjectif omniprésent dans l'expression. En effet, la sensibilité comme ensemble des impressions produites dans la relation immédiate du sujet et de la situation d'énonciation est l'une des idées les plus puissantes que développe Bally. L'idée de recourir à un critère tel que le sens « impressif » d'une forme linguistique crée l'image d'un sujet entendant idéal, qui présente toutes les facultés psychiques/psychologiques lui permettant de saisir la gradualité dans les phénomènes linguistiques par une faculté instinctive de comparaison.

Dans le Traité, le sujet psychologique, siège des facultés psychiques, est doublé d'un sujet qui assume les deux statuts distincts, celui de sujet parlant et celui de sujet entendant, appelé également interlocuteur. Le sujet est toujours le siège des facultés de l'esprit et particulièrement enclin à l'expression de son affectivité « au moment où il parle », mais ce sujet est progressivement doublé d'un sujet parlant, d'un je énonciateur, précisément par son intention d'agir sur un sujet entendant. Par exemple, la distinction entre syntaxe usuelle et syntaxe affective évoque largement la perspective du sujet parlant qui veut agir sur l'interlocuteur. Le langage expressif semble se diviser en un langage affectif et un langage actif, deux formes du « langage de la vie » correspondant aux deux perspectives mentionnées. La distinction entre les sentimaux individuels (expression de l'émotion individuelle) et les sentimaux sociaux (une forme d'affectivité sociale impliquée dans les stratégies de politesse) correspond à la même division.

En évoquant la modalité dans la définition de la phrase, Bally met en avant dans $L G L F$ une figure du sujet parlant comme source de l'énonciation et du modus. La modalité est présentée comme expression grammaticale de la partie subjective de la phrase et dans cette perspective, elle est une fonction du sujet parlant. L'affectivité n'est plus étudiée en tant que telle, son existence linguistique est la modalité, commentaire psychologique du sujet parlant à l'égard d'une représentation. La perspective est moins psychologisante, inclinant plutôt vers le pôle proprement linguistique, la catégorie de l'affectivité étant associée à la modalité.

Pour Sechehaye, le plus parfait organisme grammatical ne saurait à lui seul constituer un langage, étant seulement à la disposition du sujet parlant qui en fait usage par un acte de volonté et sous le contrôle de son intelligence. Comme Bally, il affirme la nécessité de distinguer les deux rôles, celui de sujet parlant et celui de sujet entendant. Le sujet parlant obéit aux dispositions acquises (à la grammaire), tout en cédant aux impulsions affectives du langage spontané (l'originalité se fait 
sentir dans la combinaison des éléments empruntés aux deux sources). Du côté de la compréhension, le sujet n'est que relativement passif. Entre le sens total de ce qui a été dit et le sens total de ce qui a été compris, une différence plus ou moins grande peut surgir dans l'attribution d'une valeur à chaque partie.

Une phrase est créée par une opération de synthèse et comprise par une opération d'analyse, les deux opérations n'étant pas nécessairement conformes. Cette conception des procès de production et de réception des énoncés est fondée sur la conscience de la nature créative des deux sujets de la parole. Bally avait aussi attribué au sujet entendant un rôle important, mais c'était un rôle passif. Pour Sechehaye, la position de sujet entendant suppose la mise en exercice, inconsciente, des facultés intellectuelles et de l'intuition, ce qui relève de la loi de création inconsciente au même titre que la création de la phrase par le sujet parlant. La réception est un acte de (re)création des éléments d'une grammaire, attribuée au sujet parlant grâce au phénomène de l'intuition.

Pour Frei, la linguistique devrait offrir une vue directe sur la vie du langage, mutation qui oblige le linguiste à adopter une perspective fonctionnelle sur ce qui est « correct». Est correct ce qui est conforme non pas à une norme, mais à une fonction. Dans cette perspective, la «phrase » est définie comme un jugement « destiné à » être transmis à l'entendeur, le signe, un procédé « destiné à » transmettre une signification donnée à un entendeur donné. La fonction est liée à l'intention de communication. En effet, le sujet parlant, le « parleur », n'est pas à proprement parler une subjectivité, mais une intentionnalité et le siège d'une conscience.

En ce qui concerne le « besoin d'expressivité », le recours à la notion d'intentionnalité associe ce besoin à une fonction du sujet parlant. Selon Frei, le besoin d'expressivité tend constamment à remplacer les oppositions usuelles, devenues arbitraires, par des oppositions nouvelles, censées « mettre en éveil l'attention de l'interlocuteur et [de] faire jaillir chez lui un minimum au moins de conscience » (Frei 1929, p. 302). Notons également qu'il envisage l'expressif comme une transgression volontaire, consciente du sujet parlant qui réagit à la logique et à la grammaire normatives. Les faits du langage expressif sont considérés, sous l'influence de Bally, comme un ensemble de déformations plus ou moins fortes ou plus ou moins conscientes que le « parleur » fait subir au système « normal » de la langue. 


\section{IMAGES DU SAUSSURISME}

Le deuxième point de notre réflexion concerne la/les image(s) du saussurisme qui se dégage(nt) à l'analyse comparative des publications des trois linguistes. Deux questions nous semblent significatives à ce propos. Nous tentons d'y apporter quelques éléments de réponse, utiles à la discussion sur l'identité de l'école de Genève.

\subsection{Quelles attitudes caractérisent leurs rapports au maître?}

L'analyse comparative des productions scientifiques des trois linguistes genevois entre 1900 et 1940 fait d'abord surgir une image du saussurisme qui permet de relever les caractéristiques de la première réception du CLG par les acteurs genevois ${ }^{9}$, ses premiers interprètes. Deux attitudes se manifestent dans leurs rapports au « maître » :

(i) celle qui caractérise l'ensemble de leurs articles ou fragments d'ouvrages censés appuyer ou défendre la nouveauté et l'importance des idées saussuriennes sur la linguistique générale. Cette attitude est celle de linguistes complètement engagés dans la voie du renouvellement de la linguistique sur des bases saussuriennes. De ce point de vue, les thèses du maître sont réinscrites selon la logique de la valorisation d'un élément du « patrimoine » de la linguistique. Cette attitude est caractérisée par une adhésion de principe aux idées saussuriennes (à l'héritage saussurien), reprises, diffusées, défendues, transmises comme les coordonnées d'un cadre général nécessaire à une refondation de la linguistique.

(ii) celle qui accompagne un véritable travail de réinscription des idées saussuriennes dans leurs œuvres originales, un travail que supposent la « refonte et la nouvelle disposition de ces éléments dans des théories ultérieures » (Chiss et Puech 1997, p. 10). Cette attitude se caractérise par un esprit critique motivé par les options personnelles de chaque linguiste. Elle repose plus particulièrement sur les interprétations de la dualité langue-parole et du principe de l'arbitraire du signe.

Leurs lectures personnelles des deux thèmes saussuriens favorisent le développement d'une « science de l'expression » (sous la forme de la stylistique, de la science de l'expression grammaticale, de la linguistique fonctionnelle). Il s'agit moins pourtant de concepts saussuriens réinvestis, que de discussions, d'interprétations, de diverses manières d'envisager des idées saussuriennes, qui supposent la mise en place d'un travail interprétatif important. La lecture non oppositive de la dualité langue-parole, ainsi qu'une conception nuancée du principe de l'arbitraire sont les coordonnées d'un débat nécessaire pour qu'une problématique de l'expression ait pu se développer de différentes manières, mais selon des axes

9 Sur les caractéristiques des réceptions successives du CLG, voir C. Puech (2005). 
relativement convergents ${ }^{10}$. Dans ce débat, les trois linguistes se révèlent attachés à une conception vitaliste qui semble étrangère au saussurisme.

L'image du saussurisme qui se dégage de la problématique de l'expression chez les trois linguistes permet de relever la portée et le rôle de la réinscription des thèmes saussuriens dans leurs travaux, ce qui explique partiellement la difficulté d'associer l'école de Genève au structuralisme, ainsi que la part importante d'originalité que suppose toute interprétation.

\subsection{Comment mesurer la dette envers Ferdinand de Saussure dans le développement des idées des trois linguistes?}

Chercher à mesurer la dette envers Saussure dans le développement des idées de ces linguistes est une tâche délicate. Bally et Sechehaye avaient assumé ensemble la difficile entreprise de faire connaître la pensée du maître par un livre composé d'après les notes d'étudiants, pensée que, par la suite, ils ont constamment soutenue, défendue, diffusée, continuée. Frei, quant à lui, a contribué également et avec autant de conviction à la défense de la pensée du maître. Ces trois linguistes remarquables ont développé chacun des idées originales, exploré des pistes qu'ils ont eux-mêmes créées, ouvert de nouveaux domaines d'exploration, « à l'ombre et dans la lumière du monument » (Schlanger 2008 [1992], p. 91).

Dans son ouvrage sur la mémoire des œuvres, J. Schlanger nous livre une réflexion approfondie sur le destin des "monuments » culturels. La mémoire culturelle est, affirme-t-elle, une mémoire héroïque et monumentale, une mémoire des noms propres et des œuvres (le monde culturel est foncièrement nominaliste). Même les noms collectifs, noms de genre ou d'école, motivés par les besoins de la description, de la polémique et de l'analyse, s'ajoutent aux noms propres dès lors qu'ils deviennent mémorables.

Le monument est une « réussite extraordinaire, imprévue et surplombante » (ibid., p. 90). En tant que tel, il suscite l'admiration, et organise l'avenir (il « configure la suite ») dans deux directions : d'un côté, il a un rôle incitateur et déclencheur, il « ouvre le possible », il pousse à créer, à poursuivre, à accomplir ; d'un autre côté, le chef-d'œuvre, objet d'admiration, est propre à décourager, c'est un obstacle, par sa dimension inégalable. C'est que, comme le montre J. Schlanger, « admirer rapproche et sépare » (ibid., p. 91).

La dette envers Saussure pourrait être cernée à travers les deux versants de l'admiration du «monument ». D'un côté, l'attitude manifestée est celle d'un engagement total pour la diffusion et la continuation des idées saussuriennes, qui exercent ainsi une force d'émulation d'une intensité impressionnante ${ }^{11}$. D'un autre

10 Ce sont les mêmes axes que le structuralisme est réputé avoir négligés.

11 N'est-ce pas là l'origine de toute doctrine? C. Puech (2000) fait remarquer que Sechehaye 
côté se manifeste chez ces linguistes, moins souvent et avec moins de mordant, une attitude légèrement ambivalente. Cette attitude est liée au second versant de l'admiration :

Admirer revient à se reconnaître une moindre existence et un statut marginal. La génération qui admire se pose comme celle qui vient après dans le temps, quand le meilleur est déjà là - et surtout, comme celle qui est secondaire en droit, et qui regarde le meilleur de bas en haut et du dehors. Elle voudrait (pourtant et d'autant plus) exister aussi. Donc elle invente son activité et son identité : sa carrière poétique et sa dignité. (Schlanger 2008 [1992], p. 92)

Rappelons deux moments où se manifeste cette attitude, particulièrement significatifs pour le développement des idées de Bally et de Sechehaye. C'est une attitude qui associe à une reconnaissance totale de la validité des thèses saussuriennes, la nécessité d'y apporter des aménagements/précisions/compléments, comme des manières propres d'habiter l'espace ouvert par le maître. Elle fait lire en creux l'idée que cet espace n'est pas ou n'avait pas été rempli intégralement ou d'une manière entièrement adéquate. Significativement, les deux linguistes prennent position à l'égard de la dualité langue-parole.

Dans sa leçon d'ouverture du Cours de linguistique générale du 27 octobre 1913, intitulée « Ferdinand de Saussure et l'état actuel des études linguistiques », Bally présente son interprétation de la distinction saussurienne langue-parole. Il distingue d'abord la « langue parlée en général » et la « parole », au sens (présumé) saussurien. Entre la langue et la parole, Bally insère un troisième élément, celui qui fut l'objet de prédilection de ses recherches, la langue parlée. Les arguments de cette position médiane de la langue parlée apparaissent à travers une approche critique de la dualité langue-parole. Les trois points évoqués sont les suivants : la langue et la parole ne sont pas séparées par un fossé infranchissable ; la parole prend d'assaut perpétuellement le vocabulaire usuel et la grammaire logique ; et les créations affectives, en pénétrant la langue «normale », forment le composé appelé langue parlée. Son interprétation l'amène à établir un nouvel objet, qu'il situe entre la langue, définie comme construction intellectuelle, et la parole, force quelque peu transcendante qui s'exerce sur la langue et fait surgir les créations affectives.

Cette interprétation représente sa propre vision, et lui permet à la fois de se situer par rapport au maître, auquel il rend honneur, et d'affirmer ce qu'elle a d'original. Sa légitimité a une double motivation : cette approche est respectueuse des idées saussuriennes, et elle ouvre sa propre voie, à partir de là.

L'étude linguistique des caractères affectifs et subjectifs des faits d'expression est partiellement légitimée par son intégration au même objet d'étude, la langue,

est l'un des premiers à privilégier une approche des idées saussuriennes en termes de thèses, dans son compte-rendu du $C L G$ (1917). 
mais sous un angle que Saussure n'aurait pas adopté. Pour Bally, l'étude de la langue parlée ou la stylistique vient « compléter » ou continuer l'approche saussurienne. En même temps, c'est précisément le point où réside son originalité, la valeur de sa contribution. À son sens, la stylistique a une raison d'être, d'abord parce qu'elle est intégrée au programme saussurien, et ensuite par ce qui la distingue de la pensée du maître :

En somme, je reste fidèle à la distinction saussurienne entre la langue et la parole, mais j'annexe au domaine de la langue une province qu'on a beaucoup de peine à lui attribuer : la langue parlée envisagée dans son contenu affectif et subjectif. Elle réclame une étude spéciale : c'est cette étude que j'appelle la stylistique. Un des objets de mon enseignement sera de montrer comment la stylistique s'emboîte dans la linguistique générale. (Bally 1977 [1926], p. 157)

Les références aux idées saussuriennes chez Sechehaye sont innombrables. Dans l'ensemble de ses travaux, il cherche à montrer la nouveauté et la révolution qu'elles ont marquées pour la linguistique. Son approche critique est remarquablement nuancée, constructive, témoignant d'une conscience réflexive sur laquelle les idées du maître ont eu un écho constant et puissant. De façon générale, son adhésion aux thèses saussuriennes est sans faille, comme chez son collègue, d'ailleurs, mais avec un peu plus d'engagement pour leur diffusion. Le second versant de l'admiration se manifeste également chez Sechehaye, comme en témoigne son Essai de 1926 (l'introduction et l'appendice).

L'Essai propose une application et un approfondissement des principes énoncés dans le Programme de 1908. Son point de départ est fixé par Sechehaye dans la distinction saussurienne entre la langue, institution sociale, et la parole - la « mise en action de la langue par les sujets parlants (ou écrivants) pour communiquer leur pensée dans quelque circonstance particulière » (p. 4). En tant que telle, la parole ressortit à la psychologie individuelle, alors que la langue est du ressort de la psychologie collective.

Ce souci de classement est évoqué dans l'appendice (Note sur le classement des disciplines linguistiques), notamment comme un point de séparation entre son approche et celle de Saussure. Là, Sechehaye déclare que son principe d'emboîtement et les vues qui en découlent sont en parfaite conformité avec les distinctions saussuriennes du Cours. Il fait référence plus particulièrement à la distinction entre l'étude statique des faits de langage et l'étude des évolutions, qui l'avait incité à affirmer la nécessité d'une science du prégrammatical, de l'« expression libre et spontanée antérieure à toute organisation conventionnelle ». L'idée d'envisager une science du prégrammatical qui soit impliquée nécessairement dans toute étude de la parole organisée - lui servant de base concurremment avec la linguistique statique - serait inspirée directement de la conception saussurienne de l'articulation entre la langue et la parole. Sechehaye prend position à l'égard de cette dualité 
saussurienne, en indiquant les points de divergence entre ses idées et celles de Saussure. Le $C L G$ ne propose aucun classement tiré des distinctions proposées, se concentrant plutôt sur les « relations de réciprocité » qui existent entre les divers aspects du fait linguistique. Pour Saussure, la langue existe pour la parole et elle naît aussi de la parole, vue (abstraite) qui n'avait pas abouti à établir un rapport plus précis entre les deux. L'originalité (déclarée) de Sechehaye consiste à tirer de cette abstraction - notons qu'il s'agit donc d'une construction théorique sur des bases saussuriennes - un principe de subordination et de classement, mettant la parole (sous sa forme prégrammaticale) avant la langue. Il y ajoute une distinction qu'il avait omise dans le Programme, la conception claire d'une science de la parole comme « lien nécessaire entre la connaissance des états de langue et celle des évolutions » (Sechehaye 1926, p. 220), qui permettrait une adaptation complète du système des disciplines linguistiques à son objet.

Si Bally situait en position médiane l'étude de la langue parlée, à la suite de l'interprétation de la dualité langue-parole, Sechehaye introduit un critère de classement, le principe d'emboîtement qui traverse toutes ses idées, et permet d'envisager la création d'une nouvelle science, la science de la parole (organisée), qui ferait le lien entre la linguistique synchronique et la linguistique diachronique ${ }^{12}$.

Comment mesurer la dette envers F. de Saussure dans le développement de la problématique de l'expression ? Avant tout, par l'adhésion sans faille aux thèses saussuriennes, instituées comme une sorte de code, un ensemble de lois ou de principes. Deuxièmement, ce qui est très significatif, deux des idées originales de Bally (l'objet langue parlée) et de Sechehaye (le principe d'emboîtement) naissent d'une articulation aux idées saussuriennes, dans une attitude qui mêle l'adhésion et la volonté de développer cette problématique dans une nouvelle direction. Cette attitude se manifeste à l'égard de la dualité langue-parole, qui leur inspire des pistes à suivre, grâce à leur lecture non oppositive et compréhensive ainsi que leur attachement à l'idée d'une linguistique de la parole.

\section{L'ÉCOLE DE GENÈVE AVANT 1940, ENTRE LA CONFORMITÉ ET LA VARIATION}

Les deux premiers points de cette réflexion caractérisent deux traits identitaires significatifs pour l'école de Genève avant 1940, apportant en même temps quelques éléments utiles à la définition de la notion d'école.

Examinons d'abord deux notions qu'on pourrait évoquer quand il s'agit de déterminer les particularités qui font d'une structure quelconque une école de pensée : l'identité et l'unité.

Peut-on parler de l'identité d'une école ? La notion d'identité repose sur deux idées : l'idée de géniteur et celle d'unicité. La dimension génétique de l'identité se

12 Sur cette « troisième linguistique saussurienne », voir Sechehaye 1940. 
reflète dans la reconnaissance par les membres de l'école d'une origine commune, d'un fondateur, Saussure, et de leur filiation de principe. Si du point de vue institutionnel comme sociologique cette dimension est plutôt facile à cerner, du point de vue du composant théorique de leurs travaux, elle est plus difficile à mesurer, elle est moins stable parce qu'elle se reflète dans un ensemble idéologique mouvant et évolutif. C'est dans cet ensemble idéologique qu'il faudrait déterminer les éléments qui font l'unicité de l'école. Sous cet angle, il s'agit d'étudier les constructions idéologiques des membres de l'école, qui se croisent sur quelques points, qui se développent souvent conjointement, qui évoluent et qui se déplacent, tout en demeurant distinctes et parfaitement repérables. Un trait identitaire incontestable est leur saussurisme, reflété par les deux attitudes relevées ici.

Un second trait identitaire ${ }^{13}$ tout aussi significatif pour les trois linguistes est la mise en place de la problématique de l'expression, organisée selon ces trois axes : le statut de la psychologie, l'intérêt pour l'affectivité et la volonté d'instituer le sujet dans la langue. Le plus important est que cette problématique, ce deuxième trait identitaire, ne se développe pas séparément de leur saussurisme, les deux traits sont étroitement imbriqués. Cette construction idéologique repose aussi bien sur des conceptualités saussuriennes que sur des idées étrangères à la pensée du maître. Il importe de rappeler que Bally et Sechehaye se sont formés à l'école allemande et que leur attachement à la psychologie pourrait s'expliquer partiellement par là (ce n'est plus le cas pour Frei, plus sceptique à l'égard de la psychologie). Il est tout aussi vrai que la psychologie ne les inspire pas de la même manière.

La notion d'unité est-elle plus pertinente pour définir la notion d'école ? Nous pensons qu'on peut parler d'unité à condition d'accepter que l'un et le pluriel ne s'opposent pas toujours. L'unité de leurs approches est faite d'une pluralité relativement convergente, assurée par leurs interprétations (différentes) des idées saussuriennes, ainsi que par un développement de cet ensemble équilibré et suffisamment homogène qu'est la problématique de l'expression.

Les deux notions qui nous semblent les plus appropriées pour rendre compte de cet effet d'homogénéité sont la conformité et la variation (deux notions

13 En 1958, E. Coşeriu estimait que parmi les écoles saussuriennes, l'école genevoise, par ses trois représentants, Bally, Sechehaye et Frei, est « la plus proche de la compréhension de la langue comme objet culturel », " la plus attentive aux nuances significatives et à leurs valeurs subjectives » et " la plus disposée à enregistrer et à évaluer la multiple variété "verticale" et "stylistique" de la langue ». Son trait identitaire le plus significatif est associé au développement d'une "linguistique de la parole ", " synchronie mouvante, vivante, pulsative ", à l' " attention portée aux modalités de fonctionnement de la langue, en tant que technique de l'activité de parler» (trad. 2007, édition électronique, p. 12). En 1997, dans une réflexion sur l'unité présumée de la linguistique suisse, Coşeriu identifie un trait commun sous la forme du souci pour la réalité « concrète et multiforme du langage chez les individus et dans la communauté », et caractérise cette unité comme " bipolaire et dialectique », par la « tension permanente entre les deux pôles extrêmes de l'objectivisme et du subjectivisme absolus (ou presque) » (p. 8). 
que J. Schlanger évoque largement à propos de la présence des œuvres dans la mémoire). La question la plus intéressante concerne leur rapport : variation dans la conformité ou conformité dans la variation ? Les deux, mais pas au même niveau. Leur saussurisme assure une conformité minimale, un cadre où se manifeste la variation foncière, complexe, constitutive. Concrètement, l'organisation institutionnelle est un cadre du fonctionnement de l'école comme structure. La conformité est liée à l'origine et à l'organisation externe. Comme structure institutionnelle, l'école assure une proximité essentielle, des échanges, une collaboration (le régime du co-). Le programme saussurien est un objet constant de réflexion, de discussion, de débat. Il offre de ce fait même un cadre de variation. À ce premier niveau, on peut parler de variation dans la conformité.

La question suivante est de savoir si la variation, les différences entre les représentations, les conceptions, les idées, leurs développements, est dissolvante, au point d'introduire une distance irréductible entre leurs théories. Ces approches sont-elles fondamentalement disparates ? Existe-t-il une autre conformité dans cette variation, qui soit autrement constituée que la première ? Nous avons constaté qu'une conformité de deuxième degré se met en place, en filigrane, dans le développement de la problématique de l'expression. Autour de la catégorie de l'expression se dessine un ensemble relativement cohérent de problèmes, qui organisent un champ de discussion, qui ouvrent un chantier, qui demandent à être résolus. Au niveau théorique, la conformité première qu'on a pu envisager comme un "saussurisme minimal », donc une unité problématique ${ }^{14}$, crée un cadre de variation significatif, et à l'intérieur de cette variation se met en place une nouvelle conformité, qu'on peut toujours appeler minimale, une conformité en trois points : psychologie, affectivité, sujet parlant. Ces trois domaines de représentation, dont les deux premiers ont été largement investis par Bally et Sechehaye, moins par Frei, et dont le troisième est valorisé avec autant de conviction par les trois linguistes, sont des points de vue qui se discutent et se mettent en place ensemble, sous des éclairages qui parfois se rapprochent et parfois s'éloignent. Ces trois domaines forment un objet de partage au centre de méthodes heuristiques.

14 R. Godel 1984 (1961) affirme que pour les " linguistes saussuriens » de Genève, les principes saussuriens ne sont pas des « dogmes » mais des «points de vue sur le langage » et R. Amacker $(1975$, p. 13) relève que leur « saussurisme minimum [...] ne reflète que très partiellement l'enseignement du maître ». Voir aussi R. Amacker (1976) et (2000). 


\section{CONCLUSION}

Comment caractériser enfin l'école de Genève entre 1900 et 1940 ? Une réponse possible est que les travaux des linguistes qui incarnent l'esprit d'école avant 1940 associent deux traits identitaires qui les rapprochent, deux conformités minimales, qui se croisent et se développent ensemble : leur adhésion aux idées saussuriennes et la construction de la problématique de l'expression autour de trois axes, autant de points de convergence de leurs idées vers la valorisation du sujet dans l'étude de la langue.

Pourtant, il nous semble important de rappeler que tout chez ces linguistes ne se réduit pas à ces deux traits identitaires ${ }^{15}$ et que leur intérêt pour la place du sujet dans la langue ne surgit pas indépendamment des idées de l'époque : les débats qui animent la fin du XIX $x^{e}$ siècle - la catégorie plurielle de « parlers », l'importance redécouverte de la notion de « sujet parlant » grâce à Michel Bréal, les ébauches multiples de pragmatiques et de théories des actes de langage (C. Puech 2000, 2008) -, débats qui se poursuivent, nous semble-t-il, à travers les axes de la problématique de l'expression chez les trois linguistes ${ }^{16}$.

Le plus important, nous semble-t-il, est de s'assurer que le geste réducteur que suppose le fait d'établir, à partir d'hypothèses interprétatives, la (les) conformité(s) ne soit en rien une simplification.

\section{BIBLIOGRAPHIE}

\section{Sources primaires}

Bally, Charles, 1905. Précis de stylistique : esquisse d'une méthode fondée sur l'étude du français moderne, Genève, Eggimann.

- 1909. Traité de Stylistique française, 2 vol., Heidelberg, Winter ; Paris, Klincksieck [Genève, Librairie Georg, Paris, Klincksieck, 1951].

- 1910. «Plan d'un cours de stylistique », Sur la stylistique : articles et conférences, Édité, présenté, annoté et commenté par Étienne Karabétian, Paris, Eurédit, 2007, 77-83.

- 1911. "L'étude systématique des moyens d'expression », Sur la stylistique : articles et conférences, Édité, présenté, annoté et commenté par Étienne Karabétian, Paris, Eurédit, 2007, 105-137.

- 1926, $2^{\mathrm{e}}$ édition augmentée. Le langage et la vie, Genève, Droz [3édition augmentée, Genève, Droz, 1977].

- 1914. "Figures de pensée et formes linguistiques », Sur la stylistique : articles et conférences, Édité, présenté, annoté et commenté par Étienne Karabétian, Paris, Eurédit, 2007, 220-271.

15 Il suffit de mentionner le travail de Bally dans le domaine de la didactique et de la linguistique sociologique, que $\mathrm{Cl}$. Forel a analysé dans ses articles et dans son ouvrage de 2008, qui accompagne la publication d'une masse importante de manuscrits inédits.

16 O. Amsterdamska (1987) estime que ce sont le plus souvent les innovations qui rendent possibles les continuités. Quelles sont ces continuités ? S'agit-il d'un cadre qui se poursuit ? À notre sens, il s'agit d'un ensemble idéologique qui se transmet, qui circule, qui se transforme. Les discontinuités sont des mutations d'envergure plus ou moins importante, produites sur un ou plusieurs éléments de cet ensemble idéologique. 
- 1932. Linguistique générale et linguistique française, Berne, Francke (4édition, 1965). Frei, Henri, 1929. La grammaire des fautes, Paris, P. Geuthner [Paris, Ennoïa, 2007]. Sechehaye, Charles-Albert, 1908a. "La stylistique et la linguistique théorique ", Mélanges de linguistique offerts à M. Ferdinand de Saussure, Paris, Champion [ParisGenève Slatkine Reprints, 1982, 155-187].

- 1908b. Programme et méthodes de la linguistique théorique. Psychologie du langage, Paris, Champion.

- 1917. "Les problèmes de la langue à la lumière d'une théorie nouvelle », Revue philosophique de la France et de l'Étranger 42/7, 1-30.

- 1926. Essai sur la structure logique de la phrase, Paris, Champion.

Sechehaye, Charles-Albert, 1927. "L'école genevoise de linguistique générale ", Indogermanische Forschungen 44, 217-241.

- 1940. « Les trois linguistiques saussuriennes », Vox Romanica 5, 1/2, 1-48.

Saussure, Ferdinand de, 2002. Écrits de linguistique générale, Texte établi et édité par Simon Bouquet et Rudolf Engler, Paris, Gallimard.

Textes inédits [1908-1943] de Charles Bally et d'Albert Sechehaye édités par AnneMarguerite Fryba-Reber, sous le titre « De la cohésion et de la fluidité de la langue », Cahiers Ferdinand de Saussure 54, 2001, 429-487.

\section{Bibliographie secondaire}

Amacker, René, 1975. Linguistique saussurienne, Genève, Droz.

- 1976. «L'influence de Ferdinand de Saussure et la linguistique générale d'inspiration saussurienne en Suisse (1940-1970) », Cahiers Ferdinand de Saussure 30, 71-96.

- 2000. "Le développement des idées saussuriennes chez Bally et Sechehaye », Historiographia linguistica 27, 205-264.

Amsterdamska, Olga, 1987. Schools of thought. The Development of Linguistics from Bopp to Saussure, Dordrecht/Boston/Lancaster/Tokyo, D. Reidel Publishing Company.

Auroux, Sylvain, 1998. La raison, le langage et les normes, Collection Sciences, modernités, philosophies, Paris, P.U.F.

Chiss, Jean-Louis et Puech, Christian, 1997. Fondations de la linguistique. Études d'histoire et d'épistémologie, $2^{\mathrm{e}}$ édition, Bruxelles, Duculot.

Colombat, Bernard, Fournier, Jean-Marie, Puech, Christian, 2010. Histoire des idées sur le langage et les langues, Paris, Klincksieck.

Coşeriu, Eugenio, 1958. Sincronía, diacronía e historia. El problema del cambio lingüistico. Montevideo (Facultad de Humanidades y ciencias). [Synchronie, diachronie et histoire, Traduction en 2007 par T. Verjans. Texte français intégral disponible sur www.revuetexto.net/Parutions/Parutions.html].

- 1958. Sincronía, diacronía e historia. El problema del cambio lingüistico. Montevideo (Facultad de Humanidades y ciencias). [Synchronie, diachronie et histoire, Traduction en 2007 par T. Verjans. Texte français intégral disponible sur www.revue-texto.net/ Parutions/Parutions.html].

- 1997. «Présentation » du volume Les linguistes suisses et la variation linguistique (Wüest Jakob éd.), actes du Centenaire du Séminaire des langues romanes de l'Université de Zürich, Bâle et Tübingen, Franche Verlag, 7-19.

Forel, Anne-Claire, 2008. La linguistique sociologique de Charles Bally. Étude des inédits. Genève, Droz.

Fryba-Reber, Anne-Marguerite, 2001. « La revanche de la stylistique : hommage d'Albert Sechehaye à son prédécesseur et ami Charles Bally ", Cahiers Ferdinand de Saussure 54, 125-144.

Godel, Robert, 1984 [1961]. « L'école saussurienne de Genève », Cahiers Ferdinand de Saussure 38, 77-82. 
Puech, Christian, 2000. «L'esprit de Saussure - Paris contre Genève : l'héritage saussurien », Modèles linguistiques 20, P.U.L., 79-93 [disponible en ligne sur www.unice.fr, sous le titre « L'esprit de Saussure : réception et héritage (l'héritage linguistique saussurien : Paris contre Genève) », consulté le 8 juillet 2013].

- 2005. «L'émergence de la notion de "discours" en France et les destins du saussurisme », Langages 159, Linguistique et poétique du discours. À partir de Saussure, 93-110.

Rosenthal, Victor, Visetti, Yves-Marie, 2008. " Modèles et pensées de l'expression : perspectives microgénétiques ", Intellectica 50, 177-252.

Schlanger, Judith, 1992. La mémoire des æuvres, Paris, Nathan [Éditions Verdier, Lagrasse, 2008]. 